\title{
ON A CLASS OF FUNCTIONAL DIFFERENTIAL EQUATIONS HAVING SLOWLY VARYING SOLUTIONS
}

\section{Kusano Takaŝi and Vojislav Marić}

To the memory of Professor Tatjana Ostrogorski.

\begin{abstract}
Functional differential equations with deviating arguments are studied for the first time in the framework of Karamata regularly varying functions. A sharp condition is established for the existence of slowly varying solutions for a class of second order linear equations of the form $x^{\prime \prime}=q(t) x(g(t))$, both in the retarded and in the advanced case.
\end{abstract}

\section{Introduction and results.}

The theory of regular variation, which was initiated by Karamata in 1930, has provided a major tool for various branches of mathematical analysis including Abelian and Tauberian theorems, analytic number theory and complex analysis, and it is equally important for probability theory.

We recall that a measurable function $f:[0, \infty) \rightarrow(0, \infty)$ is said to be regularly varying of index $\rho \in \mathbf{R}$ if it satisfies

$$
\lim _{t \rightarrow \infty} \frac{f(\lambda t)}{f(t)}=\lambda^{\rho} \quad \text { for any } \lambda>0 .
$$

The totality of regularly varying functions of index $\rho$ is denoted by $\operatorname{RV}(\rho)$. The symbol SV is used to denote $\mathrm{RV}(0)$ and a member of $\mathrm{SV}=\mathrm{RV}(0)$ is referred to as a slowly varying function. If $f(t) \in \operatorname{RV}(\rho)$, then $f(t)=t^{\rho} L(t)$ for some $L(t) \in \mathrm{SV}$, and so the class of slowly varying functions is of fundamental importance in regular variation. In the later part of the paper, among many basic properties of slowly varying functions, we emphasize the representation theorem which asserts that $L(t) \in \mathrm{SV}$ if and only if it is expressible in the form

$$
f(t)=c(t) \exp \left\{\int_{a}^{t} \frac{\varepsilon(s)}{s} d s\right\}, \quad t \geqslant a,
$$

2000 Mathematics Subject Classification: Primary 34K06; Secondary $26 \mathrm{~A} 12$. 
for some $a>0$ and some measurable functions $c(t)$ and $\varepsilon(t)$ such that

$$
\lim _{t \rightarrow \infty} c(t)=c_{0} \in(0, \infty) \text { and } \lim _{t \rightarrow \infty} \varepsilon(t)=0 .
$$

For the most comprehensive exposition of regular variation and its applications, the reader is referred to the book of Bingham, Goldie and Teugels [2].

The history of the study how Karamata's theory intersects with the theory of differential equations began in 1947 by the seminal paper of Avakumovic on the Thomas-Fermi equation, [1]. Linear equations were first studied by Omey in 1981, [13]. Systematic investigations in this direction started with a paper of Marić and Tomić [11] published in 1976. A complete survey of the results on differential equations, both linear and nonlinear, developed by means of regular variation is given in the monograph of Marić [10]. It is shown therein that the class of Karamata regularly varying functions is a well-suited framework for the asymptotic analysis of nonoscillatory solutions of second order linear and nonlinear differential equations. As an example for that statement we give the following theorem due to Marić and Tomić [12] (see also [10, Thm. 1.1]), which provides a sharp criterion for the existence of a slowly varying solution to the second order linear differential equation

$$
x^{\prime \prime}=q(t) x, \quad q(t)>0,
$$

where $q$ is continuous and integrable on some positive half-axis $(a, \infty)$.

THEOREM 1.1. Equation (A) possesses a slowly varying solution $x(t)$ if and only if

$$
\lim _{t \rightarrow \infty} t \int_{t}^{\infty} q(s) d s=0 .
$$

It is decreasing and can be represented in the form

$$
x(t)=x\left(t_{0}\right) \exp \left\{\int_{t_{0}}^{t} \frac{v(s)-Q(s)}{s} d s\right\}, \quad t \geqslant t_{0},
$$

for some $t_{0}>a$, where

$$
Q(t)=t \int_{t}^{\infty} q(s) d s
$$

and $v(t)$ is a solution of the integral equation

$$
v(t)=t \int_{t}^{\infty}\left(\frac{v(s)-Q(s)}{s}\right)^{2} d s, \quad t \geqslant t_{0} .
$$

Since by (1.1), $Q(t) \rightarrow 0$, as $t \rightarrow \infty$, one can choose $t_{1}>t_{0}$ so large that

$$
8 Q(t) \leqslant \theta<1, \text { for } t \geqslant t_{1} .
$$

Further study of equation (A) and its generalizations in the spirit of Theorem 1.1 has been carried out by Howard and Marić [5] and Jaroš and Kusano [6], [7].

A question naturally arises concerning the possibility of investigating the asymptotic behavior of functional differential equations with deviating arguments in the framework of Karamata functions. To the best of the authors' knowledge, nothing is known about this subject except for a paper of Grimm and Hall [3], in which 
the slowly varying character of positive decreasing solutions of some differential equations with advanced argument was discussed.

The present work was motivated by this observation and attempts to establish the existence of a slowly varying solution (an SV-solution for short) for equations of the type

$$
x^{\prime \prime}(t)=q(t) x(g(t)), \quad q(t)>0,
$$

which is a companion functional differential equation to equation (A). Here again, $q$ is continuous and integrable on some positive half-axis $\left[t_{0}, \infty\right)$.

Our result pertinent to the retarded case is the following

THEOREM 1.2. Suppose that $g:[0, \infty) \rightarrow \mathbf{R}^{+}$is a continuous increasing function such that $g(t) \rightarrow \infty$, as $t \rightarrow \infty$, satisfying $g(t)<t$, for $t \geqslant t_{0}$ where $t_{0}$ is such that $g(t) \geqslant t_{0}>1$, for $t \geqslant t_{1}$ and

$$
\int_{g(t)}^{t} \frac{Q(s)}{s} d s \leqslant 1 / e, \quad t \geqslant t_{1} .
$$

Then equation (B) possesses a slowly varying solution if and only if condition (1.1) is satisfied.

Obviously, this solution is nonoscillatory since SV-functions are positive by definition.

The result pertinent to the advanced case is the following

THEOREM 1.3. Suppose that $g:[0, \infty) \rightarrow \mathbf{R}^{+}$is a continuous increasing function such that $g(t) \rightarrow \infty$, as $t \rightarrow \infty$, satisfying $g(t)>t$, for $t \geqslant t_{1}$, and

$$
\int_{t}^{g(t)} \frac{Q(s)}{s} d s \leqslant 1 / e, \quad t \geqslant t_{1} .
$$

Then equation (B) possesses a slowly varying solution if and only if condition (1.1) is satisfied.

To establish the existence of an SV-solution for (B) we proceed as follows. First, we form an infinite family of differential equations of the form (A) each of which possesses an SV-solution, and then, with the help of the Schauder-Tychonoff fixed-point theorem, look for the equation in the family whose SV-solution exactly gives birth to the desired solution of equation (B). To make this procedure feasible we need precise information about the structure of the SV-solutions of differential equations of the form (A) without functional argument. The proof of Theorem 1.1 will be given in Section 2 for completeness, and those of Theorems 1.2 and 1.3 will be presented in Section 3.

To conclude the introduction it should be mentioned that oscillation theory of functional differential equations including equation (B) has been the subject of intensive investigations for the past three decades and that there is a vast literature devoted to the study of the oscillatory and nonoscillatory behavior of a variety of such equations from diverse angles and viewpoints. See for example the books of Györi and Ladas [4], Koplatadze and Chanturia [8] and Ladde, Lakshmikantham and Zhang [9]. 
REMARK 1.1. For the linear equation (A), there exist results of a similar nature also when the limit in (1.1) is positive, [10, Thms. 1.2 and 1.11]. It seems plausible that such results hold for equation (B) under suitable assumptions on $g(t)$. This, however will be a subject of our future investigations.

\section{Preliminaries}

The proof of Theorem 1.1 given here is elementary and follows [10]. A different one is given by Jaroš and Kusano in [6]. It makes use of a fixed-point method and is applicable also for the case when $q(t)$ is of unrestricted sign.

The "only if" part. Let $x(t)$ be an SV (hence positive) solution of equation (A). Since it is convex due to $q(t)>0$, it is monotone and in addition, because of [10, Prop. 9c], one has

$$
\lim _{t \rightarrow \infty} \frac{t x^{\prime}(t)}{x(t)}=0
$$

Write equation (A) in the form

$$
\left(x^{\prime}(t) / x(t)\right)^{\prime}+\left(x^{\prime}(t) / x(t)\right)^{2}=q(t),
$$

integrate over $(t, \infty)$, use (2.1) and multiply throughout by $t$ to obtain

$$
t x^{\prime}(t) / x(t)+t \int_{t}^{\infty}\left(s x^{\prime} / x\right)^{2} s^{-2} d s=t \int_{t}^{\infty} q(s) d s .
$$

Due to (2.1) the left-hand side integral, and so the one on the right-hand side, converge. Moreover, both sides tend to zero as $t \rightarrow \infty$.

Observe that here the convergence of the integral of $q(s)$ is a consequence, not a hypothesis.

The "if" part. It is known that equation (A) has a positive decreasing solution on $(a, \infty)$, [10, Lemma 1.1]; denote it again by $x(t)$. Then integrate on both sides of $(\mathrm{A})$ over $(t, \infty)$. Since $x(t)$ is decreasing and convex, it is such that $x^{\prime}(t) \rightarrow 0$, $t \rightarrow \infty$. This leads to

$$
-x^{\prime}(t)=\int_{t}^{\infty} q(s) x(s) d s
$$

and so

$$
0<-\frac{t x^{\prime}(t)}{x(t)} \leqslant t \int_{t}^{\infty} q(s) d s .
$$

The right-hand side tends to zero as $t \rightarrow \infty$ by hypothesis, whence $(2.1)$ follows and consequently, $x(t)$ is SV, [10, Prop. 10].

Observe that $x^{\prime}(t) \rightarrow c>0$, as $t \rightarrow \infty$ cannot hold. For, this would imply $x(t) \sim c x$ contradicting the fact that $x(t)$ decreases.

Also, an SV-solution $x(t)$ cannot increase. For otherwise, due to the convexity, one would have eventually $x^{\prime}(t) \geqslant k$, for some $k>0$, or by integrating, $x(t) \geqslant k t+l$ which is impossible for an SV function [10, Prop. 4.ii].

To obtain the representation (1.2) put

$$
\frac{x^{\prime}(t)}{x(t)}=\frac{v(t)-Q(t)}{t} \text {. }
$$


An integration over $\left(t_{0}, t\right)$ gives (1.2). Here $v(t)$ is indeed a solution of (1.4); as is well known the right-hand side of (2.2) satisfies the Riccati equation

$$
\left(\frac{v(t)-Q(t)}{t}\right)^{\prime}+\left(\frac{v(t)-Q(t)}{t}\right)^{2}=q(t)
$$

or, due to (1.3),

$$
\left(\frac{v(t)}{t}\right)^{\prime}+\left(\frac{v(t)-Q(t)}{t}\right)^{2}=0
$$

which by integrating over $(t, \infty)$, since $\frac{v(t)}{t} \rightarrow 0$ due to (2.2), gives (1.4).

Observe that in view of (1.4),v(t) is positive for $t \geqslant t_{1}$. Also since $x^{\prime}(t)<0$, $x(t)$ being decreasing, one obtains from $(2.2), v(t) \leqslant Q(t), t \geqslant t_{1}$; i.e.

$$
0<v(t) \leqslant Q(t), \quad t \geqslant t_{1}
$$

\section{Proofs}

Our purpose here is to give proofs of Theorems 1.2 and 1.3 based on Theorem 1.1

Proof of Theorem 1.2. Indeed, the "only if" part is a direct consequence of the later theorem. For, suppose that there exists an SV-solution $x(t)$ of equation (B) on $\left[t_{0}, \infty\right)$; then one writes it as

$$
x^{\prime \prime}(t)=q_{x}(t) x(t), \quad t \geqslant t_{0},
$$

where $q_{x}(t)=q(t) x(g(t)) / x(t)$. It follows, by Theorem 1.1, that $t \int_{t}^{\infty} q_{x}(s) d s \rightarrow 0$ as $t \rightarrow \infty$. This implies (1.1) since $x(g(t)) / x(t) \geqslant 1$ by the decreasing nature of $x(t)$.

The proof of the "if" part. Suppose that (1.1) holds. Let us define $\Xi$ to be the set of positive, continuous nonincreasing functions $\xi(t)$ on $\left[t_{0}, \infty\right)$ such that

$$
\xi(t)=1, \quad \text { for } t_{0} \leqslant t \leqslant t_{1}
$$

and

$$
\frac{\xi(g(t))}{\xi(t)} \leqslant e \quad \text { for } t \geqslant t_{1}
$$

$t_{1}$ being defined by (1.5). We remark that $\Xi$ is a nonvoid set since it contains e.g., nonincreasing functions $\xi_{\lambda}(t), \lambda \in(0, e]$, given by

$$
\xi_{\lambda}(t)=1, \quad t_{0} \leqslant t \leqslant t_{1}, \quad \xi_{\lambda}(t)=\exp \left\{-\lambda \int_{t_{1}}^{t} \frac{Q(s)}{s} d s\right\}, \quad t \geqslant t_{1} .
$$

To show that (3.3) also holds, notice that due to the properties of $g(t)$, there might exist an interval $t_{1} \leqslant t \leqslant t_{2}$, where $g(t) \leqslant t_{1}$ and $g(t) \geqslant t_{1}$ for $t \geqslant t_{2}$. But then, due to (3.2), inequality (3.3) holds for $t_{1} \leqslant t \leqslant t_{2}$ and for $t \geqslant t_{2}$, by (1.6), one has

$$
\frac{\xi_{\lambda}(g(t))}{\xi_{\lambda}(t)} \leqslant \exp \left\{e \int_{g(t)}^{t} \frac{Q(s)}{s} d s\right\} \leqslant e .
$$

Hence (3.3) holds for all $t \geqslant t_{1}$. 
The set $\Xi$ is a closed convex subset of the locally convex space $C\left[t_{0}, \infty\right)$ of continuous functions on $\left[t_{0}, \infty\right)$ equipped with the metric topology of uniform convergence on compact subintervals of $\left[t_{0}, \infty\right)$.

The set $\Xi$ is clearly convex in $C\left[t_{0}, \infty\right)$. It is also closed; for let $\left\{\xi_{n}\right\}$ be a sequence in $\Xi$ converging to $\eta$ as $n \rightarrow \infty$ (i.e., $\xi_{n}(t)$ converging uniformly to $\eta(t)$ as $n \rightarrow \infty)$ on compact subinterval of $\left[t_{0}, \infty\right)$. It is clear that $\eta(t)$ is continuous and to prove its positivity on $\left[t_{0}, \infty\right)$ one argues as follows: suppose on the contrary, that there exists a $T>t_{1}$ such that $\eta(t)>0$ for $t_{0} \leqslant t<T$ and $\eta(t)=0$ for $t \geqslant T$. By (3.3) one has $\xi_{n}(g(T)) \leqslant e \xi_{n}(T)$, and letting $n \rightarrow \infty$, there follows $0<\eta(g(T)) \leqslant e \eta(T)=0$ which is impossible. This also implies (3.3) for $\eta(t)$.

For each $\xi \in \Xi$ consider the second order ordinary differential equation

$$
x^{\prime \prime}=q_{\xi}(t) x,
$$

where $q_{\xi}(t)$ is given by

$$
q_{\xi}(t)=q(t) \frac{\xi(g(t))}{\xi(t)}
$$

Define

$$
Q_{\xi}(t)=t \int_{t}^{\infty} q_{\xi}(s) d s .
$$

Since, due to (3.3) and (1.1), $Q_{\xi}(t) \leqslant e Q(t)$ and so $Q_{\xi}(t) \rightarrow 0, t \rightarrow \infty$ for all $\xi \in \Xi$, Theorem 1.1 ensures that equation (3.4) possesses for every $\xi \in \Xi$, an SV-solution $x_{\xi}(t)$ expressed in the form

$$
x_{\xi}(t)=\exp \left\{\int_{t_{1}}^{t} \frac{v_{\xi}(s)-Q_{\xi}(s)}{s} d s\right\}, \quad t \geqslant t_{1},
$$

where $v_{\xi}(t)$ solves the integral equation

$$
v_{\xi}(t)=t \int_{t}^{\infty}\left(\frac{v_{\xi}(s)-Q_{\xi}(s)}{s}\right)^{2} d s, \quad t \geqslant t_{1} .
$$

We denote by $\Phi$ a mapping which associates with each $\xi \in \Xi$ the function $\Phi \xi$ defined by

$$
\Phi \xi(t)=1 \quad \text { for } t_{0} \leqslant t \leqslant t_{1}, \quad \Phi \xi(t)=x_{\xi}(t) \quad \text { for } t \geqslant t_{1} .
$$

We will look for a fixed point of $\Phi$ with the help of the Schauder-Tychonoff fixed-point theorem. For that we need to prove that $\Phi$ is a self-map on $\Xi$, the relative compactness of the set $\Phi(\Xi)$ in $C\left[t_{0}, \infty\right)$ and the continuity of the mapping $\Phi$.

For any $\xi \in \Xi$, the function $\Phi \xi(t)$ is obviously positive and nonincreasing for $t \geqslant t_{0}$.

Furthermore, due to the definition of $\Phi$, arguing as before, we conclude that to prove the property (3.3) one needs only to consider the case $g(t) \geqslant t_{1}$ which leads to

$$
\frac{\Phi \xi(g(t))}{\Phi \xi(t)} \leqslant \exp \left\{\int_{g(t)}^{t} \frac{Q_{\xi}(s)-v_{\xi}(s)}{s} d s\right\} \leqslant \exp \left\{e \int_{g(t)}^{t} \frac{Q(s)}{s} d s\right\} \leqslant e .
$$


It follows that $\Phi \xi \in \Xi$, implying that $\Phi$ is a self-map on $\Xi$.

Since $\Phi(\Xi) \subset \Xi, \Phi(\Xi)$ is locally uniformly bounded on $\left[t_{0}, \infty\right)$, and since $\xi \in \Xi$ implies

$$
0 \geqslant \frac{d}{d t} \Phi \xi(t)=\frac{d}{d t} x_{\xi}(t)=x_{\xi}(t) \frac{v_{\xi}(t)-Q_{\xi}(t)}{t} \geqslant-\frac{e Q(t)}{t}, \quad t \geqslant t_{1},
$$

$\Phi(\Xi)$ is locally equicontinuous on $\left[t_{0}, \infty\right)$. This guarantees via the Arzela-Ascoli lemma that $\Phi(\Xi)$ is relatively compact in $C\left[t_{0}, \infty\right)$.

Let $\left\{\xi_{n}\right\}$ be a sequence of functions in $\Xi$ converging to $\eta \in \Xi$ in $C\left[t_{0}, \infty\right)$. The continuity of $\Phi$ is guaranteed if it is shown that the sequence $\left\{\Phi \xi_{n}\right\}$ converges to $\Phi \eta$ in $C\left[t_{0}, \infty\right)$, or equivalently that $\left\{\Phi \xi_{n}(t)\right\}$ converges to $\Phi \eta(t)$ uniformly on compact subintervals of $\left[t_{0}, \infty\right)$. Using $(3.7)$ and the mean value theorem, bearing in mind that the integrand is negative, we have for $t \geqslant t_{1}$

$$
\begin{aligned}
\left|\Phi \xi_{n}(t)-\Phi \eta(t)\right| & =\left|x_{\xi_{n}}(t)-x_{\eta}(t)\right| \\
& =\left|\exp \left\{\int_{t_{1}}^{t} \frac{v_{\xi_{n}}(s)-Q_{\xi_{n}}(s)}{s} d s\right\}-\exp \left\{\int_{t_{1}}^{t} \frac{v_{\eta}(s)-Q_{\eta}(s)}{s} d s\right\}\right| \\
(3.9) & \leqslant \int_{t_{1}}^{t} \frac{\left|Q_{\xi_{n}}(s)-Q_{\eta}(s)\right|+\left|v_{\xi_{n}}(s)-v_{\eta}(s)\right|}{s} d s
\end{aligned}
$$

where $Q_{\xi_{n}}(t)$ and $Q_{\eta}(t)$ are defined by (3.6) and $v_{\xi_{n}}(t)$ and $v_{\eta}(t)$ are the solutions of the integral equation (3.8) with $\xi$ replaced by $\xi_{n}$ and $\eta$, respectively. Consequently, to verify the continuity of $\Phi$ in the topology of $C\left[t_{0}, \infty\right)$ it suffices to prove that the integrand of the last integral in (3.9) converges to 0 uniformly on any compact subinterval of $\left[t_{1}, \infty\right)$. Since

$$
\frac{\left|Q_{\xi_{n}}(t)-Q_{\eta}(t)\right|}{t} \leqslant \int_{t}^{\infty} q(s)\left|\frac{\xi_{n}(g(s))}{\xi_{n}(s)}-\frac{\eta(g(s))}{\eta(s)}\right| d s,
$$

an application of the Lebesgue dominated convergence theorem ensures the uniform convergence $\left|Q_{\xi_{n}}(t)-Q_{\eta}(t)\right| / t \rightarrow 0$ on $\left[t_{1}, \infty\right)$ as $n \rightarrow \infty$. To estimate $\mid v_{\xi_{n}}(t)-$ $v_{\eta}(t) \mid / t$ we proceed as follows. Using (3.8) we have

$$
\begin{array}{r}
\left|v_{\xi_{n}}(t)-v_{\eta}(t)\right|=t\left|\int_{t}^{\infty} \frac{\left(v_{\xi_{n}}(s)-Q_{\xi_{n}}(s)\right)^{2}-\left(v_{\eta}(s)-Q_{\eta}(s)\right)^{2}}{s^{2}} d s\right| \\
\leqslant t \int_{t}^{\infty} \frac{1}{s^{2}}\left[\left(\left|v_{\xi_{n}}(s)\right|+\left|v_{\eta}(s)\right|+\left|Q_{\xi_{n}}(s)\right|+\left|Q_{\eta}(s)\right|\right)\right. \\
\left.\quad \times\left(\left|v_{\xi_{n}}(s)-v_{\eta}(s)\right|+\left|Q_{\xi_{n}}(s)-Q_{\eta}(s)\right|\right)\right] d s
\end{array}
$$

for $t \geqslant t_{1}$, from which, noting that by (1.5),

$$
\left|v_{\xi_{n}}(t)\right|+\left|v_{\eta}(t)\right|+\left|Q_{\xi_{n}}(t)\right|+\left|Q_{\eta}(t)\right| \leqslant 8 Q(t) \leqslant \theta<1, \quad t \geqslant t_{1},
$$

we obtain

$$
\left|v_{\xi_{n}}(t)-v_{\eta}(t)\right| \leqslant \theta t \int_{t}^{\infty} \frac{\left|v_{\xi_{n}}(s)-v_{\eta}(s)\right|+\left|Q_{\xi_{n}}(s)-Q_{\eta}(s)\right|}{s^{2}} d s, \quad t \geqslant t_{1}
$$


For brevity we put

$$
z_{n}(t)=\int_{t}^{\infty} \frac{\left|v_{\xi_{n}}(s)-v_{\eta}(s)\right|}{s^{2}} d s
$$

Then, (3.11) can be rewritten as

$$
t z_{n}^{\prime}(t)+\theta z_{n}(t) \geqslant-\theta \int_{t}^{\infty} \frac{\left|Q_{\xi_{n}}(s)-Q_{\eta}(s)\right|}{s^{2}} d s, \quad t \geqslant t_{1},
$$

or equivalently

$$
\left(t^{\theta} z_{n}(t)\right)^{\prime} \geqslant \frac{-\theta}{t^{1-\theta}} \int_{t}^{\infty} \frac{\left|Q_{\xi_{n}}(s)-Q_{\eta}(s)\right|}{s^{2}} d s, \quad t \geqslant t_{1} .
$$

Noting that $t^{\theta} z_{n}(t) \rightarrow 0$ as $t \rightarrow \infty$ and integrating (3.13) from $t$ to $\infty$, we obtain

$$
t^{\theta} z_{n}(t) \leqslant \int_{t}^{\infty} \frac{\left|Q_{\xi_{n}}(s)-Q_{\eta}(s)\right|}{s^{2-\theta}} d s, \quad t \geqslant t_{1} .
$$

Using (3.14) in (3.11), we conclude that

$$
\begin{aligned}
\frac{\left|v_{\xi_{n}}(t)-v_{\eta}(t)\right|}{t} \leqslant & \frac{1}{t^{\theta}} \int_{t}^{\infty} \frac{\left|Q_{\xi_{n}}(s)-Q_{\eta}(s)\right|}{s^{2-\theta}} d s \\
& +\theta \int_{t}^{\infty} \frac{\left|Q_{\xi_{n}}(s)-Q_{\eta}(s)\right|}{s^{2}} d s, \quad t \geqslant t_{1} .
\end{aligned}
$$

Since the right-hand side of (3.15) converges uniformly on $\left[t_{1}, \infty\right)$ as $n \rightarrow \infty$, so does the function $\left|v_{\xi_{n}}(t)-v_{\eta}(t)\right| / t$. This, because of (3.9) and (3.10), establishes the continuity of the mapping $\Phi$.

Thus all the hypotheses of the Schauder-Tychonoff fixed-point theorem are fulfilled, and so there exists an element $\xi_{0} \in \Xi$ such that $\xi_{0}=\Phi \xi_{0}$. From the definition of $\Phi$ it follows that $\xi_{0}(t)$ satisfies the differential equation $\xi_{0}^{\prime \prime}(t)=q_{\xi}(t) \xi_{0}(t)$, for $t \geqslant t_{1}$, which because of (3.5) implies that $\xi_{0}^{\prime \prime}(t)=q(t) \xi_{0}(g(t))$ for $t \geqslant t_{1}$, that is, $\xi_{0}(t)$ is a solution of the functional differential equation (B) on $\left[t_{1}, \infty\right)$. That $\xi_{0}(t)$ is a slowly varying function follows from the fact that $\xi_{0}(t)$ coincides with $x_{\xi_{0}}(t)$ for $t \geqslant t_{1}$ which is an SV-solution of equation (3.4). This completes the proof of the "if" part of Theorem 1.2.

Proof of Theorem 1.3. The "only if" part: As before, by supposing that equation (B) written in the form (3.1) has an SV-solution on $\left[t_{0}, \infty\right)$ one concludes that $t \int_{t}^{\infty} q_{x}(s) d s \rightarrow 0$ as $t \rightarrow \infty$. Here $q_{x}(t)=q(t) x(g(t)) / x(t)$. Due to the representation (1.2) and condition (1.7) one has $x(g(t)) / x(t) \geqslant 1 / e$ for $t \geqslant t_{1}$ and condition (1.1) follows.

The proof of the "if" part. This time we define the set $\Xi$ as the set of positive, continuous, nonincreasing functions $\xi(t)$ on $\left[t_{1}, \infty\right)$ such that

$$
\frac{\xi(t)}{\xi(g(t))} \leqslant e \quad \text { for } \quad t \geqslant t_{1}
$$

The same reasoning as before shows that the set $\Xi$ is a nonvoid convex and closed subset of the locally convex space $C\left[t_{0}, \infty\right)$. 
Again, for each $\xi \in \Xi$, we consider equation (3.4) and use notations (3.5) and (3.6).

The mapping $\Phi$ is now defined as

$$
\Phi \xi(t)=x_{\xi}(t) \text { for } t \geqslant t_{1},
$$

where $x_{\xi}(t)$ is a slowly varying solution of (3.4) whose existence is guaranteed by Theorem 1.1 bearing in mind that $\xi(g(t)) / \xi(t) \leqslant 1$. It has the representation given by (3.7) and (3.8).

To show that mapping $\Phi$ fulfils the conditions of the Schauder-Tychonoff theorem one proceeds exactly as in the proof of Theorem B. This leads to the desired result.

REMARK 3.1. Observe that slowly varying solutions of equation (B) cannot increase. This is obtained exactly as for the linear case (A). Moreover, all positive decreasing solutions of equation (B) in the case $g(t) \geqslant t$, provided that these exist, are slowly varying. Indeed if $x(t)$ is such a solution, then

$$
-x^{\prime}(t)=\int_{t}^{\infty} q(s) x(g(s)) d s
$$

and so

$$
-x^{\prime}(t) \leqslant x(t) \int_{t}^{\infty} q(s) d s
$$

since $g(t) \geqslant t$ and $x(s)$ is decreasing. Hence, due to (1.1), one has $-t x^{\prime}(t) / x(t) \rightarrow 0$, as $t \rightarrow \infty$ so that $x(t)$ is slowly varying (compare: Grimm and Hall [3]).

\section{Examples and concluding remarks}

We present some examples illustrating Theorems 1.2 and 1.3.

EXAMPLE 4.1. Consider the equation

$$
x^{\prime \prime}(t)=q_{1}(t) x(\lambda t), \quad t \geqslant e,
$$

where $q_{1}(t)$ is defined by

$$
q_{1}(t)=\frac{1}{2 t^{2} \sqrt{\log t}}\left(1+\frac{1}{2 \sqrt{\log t}}+\frac{1}{2 \log t}\right) \exp (\sqrt{\log t+\log \lambda}-\sqrt{\log t}) .
$$

The condition (1.1) is satisfied for this equation since

$$
\int_{t}^{\infty} q_{1}(s) d s \sim \frac{1}{2 t \sqrt{\log t}} \text { as } t \rightarrow \infty,
$$

where the symbol $\sim$ is used to denote the asymptotic equivalence

$$
f(t) \sim g(t) \quad \text { as } t \rightarrow \infty \Leftrightarrow \lim _{t \rightarrow \infty} \frac{f(t)}{g(t)}=1 .
$$

Equation (4.1) is retarded for $0<\lambda<1$ and advanced for $\lambda>1$.

Notice also that here $g(t)=\lambda t$, satisfies the condition

$$
\limsup _{t \rightarrow \infty} \frac{t}{g(t)}=\frac{1}{\lambda} \quad \text { for } \quad 0<\lambda<1,
$$


which implies (1.6) and

$$
\limsup _{t \rightarrow \infty} \frac{g(t)}{t}=\lambda \text { for } \lambda>1,
$$

which implies (1.7).

Therefore, equation (4.1) possesses a slowly varying solution by Theorem 1.2 or 1.3. It is easy to check that $x(t)=\exp (-\sqrt{\log t})$ is one such solution.

An analogous reasoning holds for the case when in the considered equation (4.1) $x(\lambda t)$ is replaced by $x(t+\alpha)$. It is then retarded or advanced according as $\alpha<0$ or $\alpha>0$. Here the exponential factor in $q_{1}(t)$ should be replaced by $\exp \left(\left(\log (t+\alpha)^{1 / 2}\right)-\exp (\log t)^{1 / 2}\right.$ and repeat the argument.

An example of $\{q(t), g(t)\}$ satisfying (1.1) and (1.6) is given below.

EXAmple 4.2. Consider the retarded equation

$$
x^{\prime \prime}(t)=q_{2}(t) x\left(t^{\theta}\right), \quad t \geqslant 1,
$$

where $0<\theta<1$ and $q_{2}(t)$ is defined by

$$
q_{2}(t)=\frac{1}{2 t^{2} \sqrt{\log t}}\left(1+\frac{1}{2 \sqrt{\log t}}+\frac{1}{2 \log t}\right) \exp \{-(1-\sqrt{\theta}) \sqrt{\log t}\} .
$$

Since

$$
\int_{t}^{\infty} q_{2}(s) d s=o\left(\frac{1}{t(\log t)^{m}}\right) \quad \text { as } t \rightarrow \infty, \text { for any } m \in \mathbf{N},
$$

one can easily see that (1.1) and (1.6) are satisfied for the this equation, so that there exists an SV-solution of (4.3). In fact, (4.3) has such a solution $x(t)=$ $\exp (-\sqrt{\log t})$.

REMARK 4.1. For the differential equation (A) it is known from Marić and Tomic [12] that the condition (1.1) is also a necessary and sufficient condition for the existence of a regularly varying solution of index 1; see also Marić [10] and Jaroš and Kusano [6]. From this fact we conjecture that (1.1) would provide a sharp condition for a class of retarded equations of the form (B) to have positive solutions belonging to the class of regularly varying solutions of index 1 . We give below an example which might support the conjecture, but we are still far from its verification.

Example 4.3. Consider the equation

$$
x^{\prime \prime}(t)=q_{3}(t) x(t / e), \quad t \geqslant e,
$$

where

$$
q_{3}(t)=\frac{1}{2 t^{2} \sqrt{\log t}}\left(1+\frac{1}{2 \sqrt{\log t}}-\frac{1}{2 \log t}\right) \exp (\sqrt{\log t}-\sqrt{\log t-1}) .
$$

Since $q_{3}(t)$ satisfies (1.1) and (4.2a), the equation (4.4) possesses an SV-solution $x_{0}(t)$ by Theorem 1.2. A simple calculation shows that this equation has also the solution $x_{1}(t)=t \exp (\sqrt{\log t})$ which is a regularly varying function of index 1 . 
EXAMPLE 4.4. Consider the equation

$$
x^{\prime \prime}(t)=\exp (-(1-\gamma) t) x(\gamma t), \quad 0<\gamma<1,
$$

with $q(t)=\exp (-(1-\gamma) t)$ and $g(t)=\gamma t$ satisfying (1.1) and (4.2a). Theorem 1.2 ensures the existence of an SV-solution $x_{0}(t)$ for (4.5). One sees that (4.5) has another solution $x_{1}(t)=\exp (-t)$. Note that $\exp (-t)$ is not a regularly varying function but is a rapidly varying one of index $-\infty$.

Acknowledgment. The authors are indebted to the reviewer for several valuable comments.

\section{References}

[1] V. G. Avakumović, Sur l'équation différentielle de Thomas-Fermi, Publ. Inst. Math. Beograd 1 (1947), 101-113.

[2] N.H. Bingham, C. M. Goldie and J.L. Teugels, Regular Variation, Encyclopedia of Mathematics and its Applications 27, Cambridge University Press, 1987.

[3] L. J. Grimm and L. M. Hall, Self-adjoint differential equations and Karamata functions, in: D. Bainov and V. Covachev (Eds.) Proc. Coll. Differential Equations, VSP, Utrecht, 1993, $77-87$.

[4] I. Györi and G. Ladas, Oscillation Theory of Delay Differential Equations, Oxford Science Publications, Clarendon Press, Oxford, 1991.

[5] H. C. Howard and V. Marić, Regularity and nonoscillation of solutions of second order linear differential equations, Bull. Acad. Serbe Sci. Arts, Classe Sci. Nat. Sci. Math. 114(22) (1997), $85-98$.

[6] J. Jaroš and T. Kusano, Remarks on the existence of regularly varying solutions for second order linear differential equations, Publ. Inst. Math. (Beograd) 72(86) (2002), 113-118.

[7] J. Jaroš and T. Kusano, Self-adjoint differential equations and generalized Karamata functions, Bull. Acad. Serbe Sci. Arts, Classe Sci. Mat. Nat. Sci. Math. 129(29) (2004), 25-60.

[8] R. G. Koplatadze and T. A. Chanturia, On Oscillatory Properties of Differential Equations with Deviating Arguments, Tbilisi University Press, Tbilisi, 1977. (in Russian)

[9] G. S. Ladde, V. Lakshmikantham and B. G. Zhang, Oscillation Theory of Differential Equations with Deviating Arguments, Marcel Dekker, New York, 1987.

[10] V. Marić, Regular Variation and Differential Equations, Lecture Notes in Mathematics 1726 Springer-Verlag, Berlin, 2000.

[11] V. Marić and M. Tomić, Asymptotic properties of the solutions of the equation $y^{\prime \prime}=f(x) \phi(y)$, Math. Z. 149 (1976), 261-266.

[12] V. Marić and M. Tomić, A classification of solutions of second order linear differential equations by means of regularly varying functions, Publ. Inst. Math. (Beograd), 48(62) (1990), 199-207.

[13] E. Omey, Regular variation and its applications to second order linear differential equations, Bull. Soc. Math. Belg. 32 (1981), 207-229.

Department of Applied Mathematics

(Received 3003 2006)

Faculty of Science

(Revised 1610 2006)

Fukuoka University

Fukuoka, 841-0180, Japan

tkusano@cis.fukuoka-u.ac.jp

Serbian Academy of Sciences and Arts

Kneza Mihaila 35

11000 Beograd, Serbia

vojam@uns.ns.ac.yu 